\title{
O comportamento inovador e os efeitos sobre a produção pesqueira industrial dos estados do Pará e Santa Catarina (Brasil) e da Galícia espanhola
}

\author{
José Nazareno Araújo dos Santos* \\ Ana Paula Vidal Bastos*** \\ Gisalda Carvalho Filgueiras ****
}

\begin{abstract}
Resumo
Neste artigo, teve-se como propósito mostrar os resultados obtidos em um estudo comparativo da atividade pesqueira industrial, realizado nos dois principais estados produtores de pescado do Brasil - Santa Catarina (Sul) e Pará (Norte) - e na Região da Galícia, maior entreposto pesqueiro da Espanha. O objetivo principal é evidenciar as práticas inovativas das empresas do setor, principalmente no que tange ao seu papel acerca da promoção do desenvolvimento da atividade e seus desdobramentos sobre os respectivos ambientes. Essa possibilidade foi concretizada utilizando-se do instrumental estatístico da análise fatorial, que permitiu elaborar um indicador de desempenho inovador das empresas pesquisadas, hierarquizando-as de modo, a saber, quais as mais ou menos inovadoras. Os resultados apontaram as empresas galegas em seu conjunto como as que mais inovam, refletindo no comportamento de mercado. Desse modo, é interessante perceber o que as fazem ter um desempenho melhor em relação às brasileiras, a fim de se entender porque essas se encontram em situação não muito favorável quanto à competitividade e à sustentabilidade.
\end{abstract}

Palavras-chave: Inovação. Pesca industrial. Competitividade. Economia pesqueira. Instituições.

* Doutor em Desenvolvimento Socioambiental pelo Núcleo de Altos Estudos Amazônicos (NAEA da Universidade Federal do Pará. Professor adjunto da Faculdade de Ciências Econômicas do Instituto de Ciências Sociais Aplicadas da Universidade Federal do Pará. Pesquisador áreas de economia da inovação, economia pesqueira e economia regional e no projeto de pesquisa acadêmica na área de economia pesqueira e da inovação em cooperação com a Universidade de Santiago de Compostela, Espanha. E-mail: jonaz@ufpa.br

** Doutora em Filosofia e Economia pela Universidade de Tsukuba, Japão. Professora e pesquisadora do Núcleo de Altos Estudos Amazônicos. Coordenadora de projetos de pesquisa acadêmica na área de economia da inovação e pesca em parceria com a Universidade de Santiago de Compostela, Espanha. Pesquisadora/colaboradora no projeto Urbis/Amazônica, coordenado pelo Inpe/MCT. Vice-coordenadora do Núcleo de Altos Estudos Amazônicos, responsável pela área de pesquisa. E-mail: pbastos@ufpa.br

**** Doutora em Ciências Agrárias pela Universidade Federal Rural da Amazônia (UFRA). Professora adjunto IV da Universidade Federal do Pará, no Instituto de Ciências Sociais Aplicadas, curso de Economia e PPGE. Pesquisadora em desenvolvimento rural sustentável, pesca e economia agrícola. E-mail: gfilgueiras@ufpa.br

http://dx.doi.org/10.5335/rtee.v21i44.5358

Submissão: 15/09/2014. Aceite: 02/03/2015. 


\section{Introdução}

Historicamente, a pesca tem estreita relação com a inovação. Com o passar dos anos, em função das necessidades individuais e comerciais, a forma e a intensidade de captura dos recursos diretamente extraídos da natureza tornam a atividade cada vez menos sustentável, principalmente sob o ponto de vista ambiental e social (SACHS, 2008).

Em razão da própria característica do setor produtivo, a maior parcela de matéria-prima é obtida pelo método extrativista, e é cada vez mais evidente a necessidade de desenvolvimento de novas técnicas que permitam um melhor aproveitamento do pescado, de modo que sua sustentabilidade econômica mantenha-se viável em relação ao nível dos investimentos realizados.

Todavia, é importante salientar que na pesca, tanto quanto nas demais atividades econômicas, os processos de crescimento não acontecem de forma ilimitada, havendo, assim, constante necessidade de se implementar processos inovativos e sustentáveis. Desse modo, é fundamental a existência de investimentos em Pesquisa e Desenvolvimento (P\&D), a fim de que as inovações aconteçam de forma contínua, ainda que sejam adaptativas ${ }^{1}$ (KIM, 2005). De tal modo, a transferência tecnológica, que é um dos mecanismos do processo de sua difusão, pode ocorrer de forma mais eficaz, permitindo não somente a redução da defasagem tecnológica em relação aos locais mais desenvolvidos tecnologicamente, mas também possibilitando melhor uso dos recursos pesqueiros disponíveis, isto é, exploração sustentável (KIM, 2005; SUCASAS, 2011).

No entanto, na prática, nem todo processo de transferência de tecnologia representa efetiva garantia de sucesso em sua implementação. Em geral, sua eficácia depende da forma como tal processo é conduzido e como o ambiente, para qual será direcionada tal ação, está estruturado. O nível de P\&D local, o nível local de capacitação dos agentes, a disponibilidade adequada de recursos e o nível de integração institucional devem estar formatados para maximizar a absorção das novas tecnologias a serem experimentadas (KIM, 2005).

Por esse motivo, alguns locais destacam-se mais em relação a outros. Por exemplo, no âmbito da pesca, os ambientes em que ocorrem os processos de transferência de tecnologia, geralmente apresentam, em termos de produtividade, melhores resultados, embora em termos de sustentabilidade não se possa afirmar sua eficácia. Desse modo, a incorporação da nova tecnologia deve estar condicionada ao transbordamento da eficiência econômica para a ambiental e a social. 
Nesse contexto, o presente artigo teve como objetivo principal analisar o comportamento inovador das empresas de pesca industrial em três ambientes distintos. Trata-se dos estados brasileiros de Santa Catarina (Sul) e Pará (Norte), nessa ordem, maiores produtores nacionais de pescado em volume, e da Galícia espanhola, principal entreposto pesqueiro da Espanha. Embora sejam países diferentes, apresentam importantes pontos de convergência no âmbito da atividade, que foram fundamentais para o desenvolvimento desta pesquisa.

Além disso, a pesca é uma importante atividade socioeconômica nessas regiões e contribui diretamente para seus processos de desenvolvimento. Como a inovação é o elemento-chave para a promoção do desenvolvimento - esta é a convicção defendida neste artigo - foi esse aspecto que norteou esta pesquisa. Procurou-se identificar como efetivamente a inovação colabora com a atividade nesses respectivos ambientes. Portanto, pretende-se responder o que determina o desenvolvimento e a adoção de inovações pelos empresários da pesca nos ambientes a serem pesquisados (Pará e Santa Catarina, no Brasil, e Galícia na Espanha) e os efeitos disso sobre a competitividade das empresas.

Tanto em Santa Catarina como no Pará, no Brasil, quanto na Galícia espanhola, buscou-se compreender o ambiente externo que circunda a atmosfera da produção pesqueira, por exemplo, o ambiente institucional, uma vez que ele tem elevada relevância no processo de desenvolvimento da atividade, criando um ambiente favorável às práticas inovadoras, embora esse não seja o foco principal desta discussão.

Portanto, a compreensão de importantes processos que possibilitem a incorporação e/ou adaptação de novas formas de produção e organização produtiva, a fim de tornar menos insustentável e mais competitiva a atividade pesqueira no Brasil, foi a principal motivação do presente estudo, pois a pesca tem um importante papel no conjunto de ações que englobam a promoção do desenvolvimento do país.

Com o objetivo de estruturar a ideia central da importância da inovação no processo de desenvolvimento da pesca industrial, dividiu-se o artigo em quatro seções, além da introdução. Sequencialmente teremos uma breve apresentação teórica, a metodologia adotada, a apresentação e discussão dos resultados e as respectivas considerações finais. 


\section{A inovação no enfoque evolucionário: breves referências}

A principal discussão do escopo teórico neoschumpeteriano é o papel exercido pela inovação tecnológica e como o seu processo de mudança acaba influenciando na dinâmica da atividade econômica capitalista (POSSAS, 1989; ROSENBERG, 2006), processo que ocorre desde os anos 1970.

Buscando compreender os desdobramentos da inovação sobre o crescimento e o desenvolvimento de ambientes e agentes, tal corrente de pensamento construiu sua argumentação com base no pressuposto de que: i) a dinâmica econômica é fruto senão do intenso processo baseado em inovações, que rompe constantemente a tradicionalidade; ii) o crescimento econômico é dependente de trajetórias (econômicas, sociais, políticas); iii) as rotinas substituem o comportamento maximizador dos agentes econômicos, estabelecendo novo padrão comportamental das firmas; iv) a estática dá lugar à dinâmica (DOSI et al., 1988; LUNDVALL, 1992; NELSON; WINTER, 2005; NELSON, 2006).

Assim sendo, a visão neoschumpeteriana diferencia-se da ortodoxia, principalmente, porque tem o crescimento econômico como fruto de um processo evolutivo e dinâmico, no qual o progresso tecnológico é sua mola propulsora e a inovação é o fator-chave para que isso ocorra. Desse modo, o processo de desenvolvimento e/ou adoção de inovação é endógeno, portanto, construído internamente no ambiente da produção.

Em uma perspectiva mais microeconômica - foco deste artigo -, essa abordagem atribui especial atenção à trajetória tecnológica e ao próprio comportamento das empresas no processo de mudança econômica, em que a inovação é a base desse processo (NELSON; WINTER, 2005; TEECE, 2005). Assim, é importante destacar o papel das firmas na promoção do desenvolvimento.

Conforme destacam Nelson e Winter (2005), em virtude da ausência de equilíbrio, o desempenho das firmas determina o crescimento econômico, porque a existência de assimetria garante a dinâmica necessária para que a competitividade seja saudável, de tal modo que continuamente se tenha inovação.

Nesse sentido, as empresas, com suas competências e aptidões, são fundamentais para o processo de desenvolvimento (TEECE, 2005), pois qualitativamente conseguem interferir nos mercados e na própria trajetória de expansão bem como da economia como um todo. Seus processos internos refletem sua postura competitiva, que também é composta pela coerência tanto desses processos internos como 
dos externos, pelos incentivos recebidos e pelo ambiente que as cercam, com suas políticas e regulações (TEECE, 2005, p. 156).

Nos mais diversos ambientes de produção, embora com distinções significativas - assimetrias -, essas questões são recorrentes, quando as empresas têm bem definidos seus processos internos (organizacionais), quando suas estratégias são pautadas em ações direcionadas à competitividade e à sustentabilidade da atividade, tornam-se o próprio motor do processo de desenvolvimento econômico (TEECE, 2005).

Assim, as rotinas, em substituição ao equilíbrio estático, passam a governar os comportamentos regulares das firmas (NELSON; WINTER, 2005), tornando o processo dinâmico, ou seja, a cumulatividade orienta o processo de decisão e passa-se a ter um mecanismo de busca e seleção no qual as empresas podem melhorar seu desempenho. As rotinas, na visão de Nelson e Winter (2005), são consideradas como processos importantes na compreensão da conduta das empresas em um modelo evolutivo, haja vista que, aliadas a fatores estocásticos, acabam por determinar a própria mudança dessas práticas referentes ao comportamento das empresas.

Esse esforço que as firmas fazem no sentido da inovação, de alteração de suas rotinas, é o que os autores denominam de processo de busca de oportunidades presentes ou futuras, dado o contexto tecnológico, pois “[...] o resultado das buscas de hoje constitui tanto uma nova tecnologia bem-sucedida como um novo ponto de partida natural para as buscas de amanhã" (NELSON; WINTER, 2005, p. 373).

$\mathrm{O}$ mecanismo de seleção considera três elementos como sendo relevantes para sua efetivação. Tais elementos referem-se ao nível de lucratividade considerado adequado à inovação para as empresas do setor, à influência exercida pelo mercado (consumidores) e pelas instituições (dispositivos regulatórios existentes) e aos processos de investimento e imitação. Assim, reforçam os autores:

As inovações bem-sucedidas levam tanto a um lucro mais alto para o inovador como a lucrativas oportunidades de investimento. Portanto, as firmas crescem e, ao fazê-lo, desviam mercados das não-inovadoras e reduzem sua lucratividade, o que, por sua vez, o que levam as forças a se contraírem. Tanto os lucros visíveis das empresas inovadoras como as perdas experimentadas pelas que ficaram defasadas estimulam essas últimas a tentar imitar as primeiras (NELSON; WINTER, 2005, p. 386-387).

Isso reforça o mecanismo de seleção das inovações ao mesmo tempo que, dado o ambiente de incertezas, condiciona as expectativas de lucratividade das empresas às trajetórias naturais em andamento, cujo processo de aprendizado continuado e o respectivo acúmulo de conhecimento acabam por implicar, em virtude dos ganhos obtidos ao longo do tempo, uma situação em que os retornos do investimento em inovação sejam positivos e se transformem em uma opção mais vantajosa.

Teoria e Evidência Econômica - Ano 21, n. 44, p. 149-164, jan./jun. 2015 
Assim, o processo de seleção, juntamente com o de busca, acaba por condicionar o progresso tecnológico, sendo a difusão elemento basilar do primeiro e, sob esse âmbito, é vista como fortemente condicionada pelo contexto estrutural e institucional que a cerca e inseparável do processo de seleção. Assim, os mercados tornam-se instrumentos de operações institucionais e a capacidade de inovar conduz à evolução do progresso inovador.

Nesse aspecto, as assimetrias assumem importante papel no processo de dinâmica industrial, pois permitem que haja um processo contínuo de inovação, no qual a concorrência, aliada à criação e à transformação das estruturas industriais, acaba por induzir a um mecanismo endógeno de geração de dinâmica tecnológica por parte das indústrias no interior das estruturas de mercados industriais (KIM, 2005; NELSON; WINTER, 2005; DOSI, 2006).

Dosi (2006) também destaca como aspecto importante e útil na compreensão do progresso técnico, a trajetória tecnológica, que tem na cumulatividade (NELSON; WINTER, 2005) a força motriz desse progresso, o que permite ampliar a probabilidade de avanços subsequentes da unidade em questão - uma firma ou mesmo um país (POSSAS, 1989).

No âmbito da atividade pesqueira essas questões, em função da complexidade e particularidade da atividade, principalmente em espaços como o do Brasil, com sua diversidade cultural e produtiva, são de mais difícil visualização e compreensão, uma vez que a teoria evolucionária trata a inovação de tal modo que atividades orientadas por outro padrão de produção (em que a inovação não é estratégia formalizada) ficam fora do eixo de análise. Na região da Galícia espanhola, porém, é possível ter essa percepção.

Mas, vale destacar, existe um esforço no sentido de reformular/adaptar conceitos (LASTRES et al. 1999) e buscar inferir elementos que provem que a inovação, ainda que em uma escala diferenciada daquela recorrente nos grandes empreendimentos, é fundamental para a sustentabilidade da atividade e do próprio empreendimento ao longo do tempo. Nas seções seguintes, revelam-se os resultados mais importantes obtidos na pesquisa e os métodos utilizados.

\section{Ambientes e agentes estudados e procedimentos metodológicos adotados}

Para a construção do indicador de desempenho inovativo (IDI) das empresas de pesca do setor industrial, foram coletados dados primários obtidos diretamente nas unidades de produção. Foram entrevistados representantes de empresas em 
Santa Catarina e no Pará, no Brasil, e da região da Galícia espanhola. As informações coletadas foram orientadas a partir da literatura evolucionária e de alguns estudos acerca da inovação, como é o caso do estudo de Stallivieri e Britto (2009), fazendo-se as devidas adaptações para a atividade pesqueira.

Ao todo, foram feitas 33 entrevistas, onze em Santa Catarina, doze no Pará e dez na Galícia espanhola. Como condição de participação, estabeleceu-se que a unidade produtiva deveria fazer parte do elo industrial (beneficiamento) da cadeia produtiva do pescado. Em razão disso, tratou-se de uma amostra intencional, pois essas empresas ainda são restritas, em termos quantitativos. Como se pretendeu estabelecer comparações entre os ambientes produtivos pesquisados, houve a necessidade de se adotar um procedimento metodológico que fosse capaz de atender aos objetivos do estudo. Como já mencionado, a escolha dos locais de pesquisa deu-se em razão de sua importância produtiva e do papel da inovação. A opção pelo setor industrial de beneficiamento seguiu esse mesmo critério, pois nele a intensidade inovativa é maior e, portanto, mais representativa para os objetivos da pesquisa, ressalvando-se que, no tocante ao número de empresas, a decisão foi coerente com as exigências do modelo estatístico utilizado bem como com as características desejáveis a fim de haver uma maior representatividade do segmento.

Nesse sentido, o uso de indicadores mostrou-se mais viável. Assim, adotou-se o indicador de desempenho, que permite comparar as unidades produtivas por meio da hierarquização, isto é, as empresas são classificadas de modo decrescente em função de sua performance inovadora. Para sua construção utilizou-se a técnica estatística multivariada de análise fatorial, que é apresentada, na sequência, de forma resumida.

\subsection{Variáveis utilizadas na construção do IDI}

Nesta seção são apresentadas as variáveis que compõem o modelo fatorial do indicador de desempenho inovador das empresas de pesca que foram analisadas para a pesquisa, no Brasil e na Espanha. Em razão da necessidade de se ajustar o modelo, o uso das informações obtidas ficou limitado às seguintes:

i) tipo de inovação (processo ou produto);

ii) frequência de inovação (intensidade de uso ou de desenvolvimento e/ou adoção de inovações); 
iii) tipo de insumo utilizado no produto novo (identificar se a matéria-prima usada era o produto básico e/ou resíduo da produção);

iv) grau de inovação (identificar qual o nível de inovação adotado - baixo, médio ou alto);

v) origem da inovação (faz referência ao desenvolvimento ou não interno de inovação);

vi) indicador de qualificação (refere-se à proporção do nível de formação superior ou pós-graduação em relação ao total de trabalhadores agregados nas empresas entrevistadas);

vii) produtos lançados no biênio (quantidade de produtos novos lançados nos últimos dois anos).

\subsection{A análise fatorial e o IDI}

O modelo de análise fatorial de análise doscomponentes principais apresenta-se da seguinte forma:

$$
\begin{aligned}
C P 1= & \gamma 11 X 1+\gamma 12 X 2+\ldots+\gamma 1 P X p \\
C P 2= & \gamma 21 X 1+\gamma 22 X 2+\ldots+\gamma 2 P X p \\
& \ldots \ldots \ldots \ldots \ldots \ldots \ldots \ldots \ldots \ldots \ldots \ldots \ldots \ldots \ldots \ldots \ldots
\end{aligned}
$$

De acordo com Fávero et al. (2009), a partir da padronização de X (média 0 e desvio padrão 1), o modelo fatorial passa a ser escrito, genericamente, da seguinte forma:

$$
X_{i}=A_{i 1} F_{1}+A_{i 2} F_{2}+\cdots A_{i k} F_{k}+E_{i}
$$

Em que:

$X_{i}=$ são as i-ésimas variáveis $(\mathrm{i}=1,2, \ldots, \mathrm{p})$;

$\mathrm{F}_{1}, \mathrm{~F}_{2}, \ldots, \mathrm{F}_{\mathrm{k}}=$ são fatores extraídos;

$\mathrm{A}_{\mathrm{i}}=$ são as cargas fatoriais (sendo $\mathrm{i}=1 . ., \mathrm{k}$ );

$\varepsilon_{i}=$ são os i-ésimos fatores únicos.

Após a obtenção dos fatores, quando as variáveis são agrupadas em fatores a partir da magnitude de suas cargas fatoriais, o fator pode ser definido da seguinte forma: 


$$
F_{j}=\lambda_{j 1} X_{1}+\lambda_{j 2} X_{2}+\lambda_{j 3} X_{3}+\ldots,+\lambda_{j n} X_{n}
$$

Sendo:

$\mathrm{F}_{\mathrm{j}}$ : i-ésimo fator;

$\lambda_{\mathrm{ji}}$ : são os coeficientes dos escores fatoriais;

$\mathrm{X}_{\mathrm{n}}$ : são as n observações das $\mathrm{X}$ variáveis possíveis;

n: é o número de variáveis.

A partir desses desdobramentos estatísticos pode-se constituir o indicador de desempenho inovativo, que é definido como uma combinação linear dos escores fatoriais obtidos por meio da análise fatorial, e a proporção da variância é explicada pelo fator em relação à variância total (SANTANA; CARVALHO; MENDES, 2008, p. 126). Por meio desse indicador é possível hierarquizar as empresas em função da importância atribuída à inovação. Esse indicador apresenta uma escala de classificação na qual se enquadra o desempenho inovador das firmas como alto $(\leq 0,700$ $\leq 1)$, médio $(\leq 0,350 \leq 0,699)$ e baixo $(\leq 0 \leq 0,349)$ (SANTANA; CARVALHO; MENDES, 2008). Matematicamente o indicador é apresentado sob a expressão:

$$
I D I=\sum_{j=1}^{\mathrm{q}}\left(\frac{\lambda j}{\sum \lambda j} F P i j\right)
$$

Sendo que:

$\lambda$ : é a variância explicada por cada fator individualmente;

$\Sigma \lambda$ : é a soma total da variância explicada pelo conjunto de fatores comuns;

FP: é o escore fatorial padronizado, ou seja, é o procedimento realizado para tornar os escores originais positivos a fim de permitir a hierarquização das empresas.

O FP tem valores compreendidos no intervalo de 0 a 1 . Sua expressão matemática é:

$$
F P I=\left(\frac{F_{i}-F_{\text {min }}}{F_{\text {max }}-F_{\text {min }}}\right)
$$

Em que:

$\mathrm{F}_{\mathrm{i}}$ : é o valor do escore fatorial a ser padronizado;

$\mathrm{F}_{\text {min }}$ : é o valor mínimo observado para o conjunto de escores fatoriais extraídos;

$\mathrm{F}_{\max }$ : é o valor máximo observado para o conjunto de escores fatoriais extraídos. 
Seguindo a orientação de Santana, Carvalho e Mendes (2008), com o objetivo de facilitar a compreensão dos resultados e a própria hierarquização das empresas de pesca em função do IDI, estabeleceram-se os seguintes intervalos de valores: IDI igual ou superior a 0,70 são considerados altos; entre 0,35 e 0,69, intermediários; e inferiores a 0,35 , baixos ${ }^{2}$.

\section{Resultados e discussões}

\subsection{Aspectos gerais}

Em termos gerais, os ambientes pesquisados apresentaram pontos de convergência e de divergência no que se refere à adoção e/ou à forma de inovação adotada no processo de produção da pesca industrial. $O$ primeiro ponto de convergência a ser apontado diz respeito ao tipo de inovação adotado. Tanto no Pará quanto em Santa Catarina, no Brasil, e na Galícia espanhola, as empresas de pesca industrial apresentaram inovação de processo e de produto.

No que concerne à frequência, as empresas espanholas apresentaram melhor desempenho, $100 \%$ delas realizaram algum tipo de inovação. Um terço inovou em processo e as demais em processo e produto. Em seguida, as empresas paraenses, com $92 \%$, e as catarinenses, com $91 \%$. Do total das empresas inovadoras paraenses, não menos que $64 \%$ inovou em processo e produto, e $9 \%$ somente em produto. As catarinenses apresentaram, respectivamente, valores relativos da ordem de $70 \%$ e $20 \%$.

Quando se analisa a categoria produtos, identifica-se que os mais elaborados (industrializados e/ou prontos imediatamente para consumo) são desenvolvidos pelas empresas galegas. Setenta por cento dos novos produtos lançados por essas empresas nos últimos dois anos agregam-se nesse grupo e apenas $8 \%$ estão na categoria modificado. As empresas de Santa Catarina apresentaram resultado intermediário, isto é, $57 \%$ de seus produtos lançados no último biênio foram elaborados e 19\%, modificados. De certo modo, essa condição pode ser justificada pela presença de empresas espanholas atuando diretamente no complexo produtivo pesqueiro de Santa Catarina. Diferentemente, as empresas paraenses apresentaram o mais fraco desempenho. Do total de produtos lançados no período considerado, $74 \%$ foi modificado e somente $4 \%$ elaborado.

Quando questionados acerca da finalidade das inovações, as repostas foram bem diversas em relação às empresas dos diferentes ambientes pesquisados. Por exemplo, as empresas do norte do Brasil (Pará) apontaram que 8\% significa uma 
questão estratégica propriamente dita. As demais firmas inovaram para atender a uma demanda não tão específica, como é o caso da legislação. Quando se analisam os dados das empresas catarinenses, os resultados são surpreendentes. Não menos que 55\% das empresas que inovam, o fazem por uma questão exclusivamente de estratégia, dado superior às empresas galegas (Espanha), essas, com ordem de 30\%.

É destacável em relação a essas informações que no tocante a um aspecto mais amplo de análise, as empresas da Galícia espanhola apresentam-se com melhores resultados, pois quando analisada em conjunto com o desempenho, a finalidade da inovação atinge o índice de $80 \%$, superior ao conjunto das firmas dos dois demais ambientes pesquisados. Desse modo, os resultados apresentados na seção seguinte justificam-se também em termos das variáveis que não estão inseridas no modelo de análise fatorial.

\subsection{Análise fatorial}

Os resultados estatísticos do modelo de análise fatorial, com base nos dados trabalhados, mostraram-se satisfatórios. Os testes de adequação do modelo (Bartlett e KMO) apresentaram significância estatística a 1\% de probabilidade de erro, portanto, com alto grau de confiabilidade.

Os dados de Santa Catarina apresentaram valor da ordem de 47,8 para o teste de Bartlett, enquanto que Galícia foi da ordem de 72,5, indicando que mesmo havendo forte correlação entre as variáveis, a matriz de correlação não é identidade. No que se refere ao resultado do KMO, os dados da Galícia apresentaram razoabilidade de adequação dos dados. O valor do referido teste foi 0,536.

Para Santa Catarina, o valor foi de 0,758, o que significa um alto grau de adequação dos dados. Assim, os valores são aceitáveis. Considerando-se os dois testes, pode-se então afirmar que o uso da técnica da análise fatorial para a obtenção do IDI é apropriado e deve-se prosseguir com o modelo.

Foram extraídos para a composição do índice de desempenho inovador das empresas dois fatores, de sete possíveis, os quais, para cada conjunto de dados, apresentam nível distinto de explicação. Por exemplo, os fatores extraídos para os dados de Santa Catarina explicam 78,38\% do total da variância, enquanto que para a Galícia espanhola, esse percentual é de 84,491. Logo, todos têm elevado poder explicativo. Na seção a seguir, são apresentados os resultados do IDI das empresas pesquisadas por ambiente e os prováveis fatores que justificam a classificação. 


\subsection{O índice de desempenho inovador das empresas de pesca industrial pesquisadas: fatos destacáveis}

O indicador de desempenho inovador constituído com os dados das empresas de pesca industrial de Santa Catarina e Pará (Brasil), e da região da Galícia (Espanha), mostrado sequencialmente foi constituído pelas informações das seguintes variáveis: frequência de inovação, tipo de inovação, grau de inovação, origem da inovação, nível de qualificação da mão de obra empregada, tipo de insumo utilizado na produção de novos produtos e quantidade de produtos novos comercializados.

Com tais variáveis, o modelo ajustou-se, permitindo realizar o processo de hierarquização das unidades pesquisadas bem como estabelecer comparações entre os ambientes. Conforme é demonstrado pelos dados e pela classificação sugerida por Santana, Carvalho e Mendes (2008), as empresas galegas apresentaram, em um computo geral, melhor desempenho do que as brasileiras (Tabela 1).

Tabela 1 - Resultado do índice de desempenho inovador das empresas de pesca industrial de SC e PA (Brasil) e da Galícia (Espanha)

\begin{tabular}{l|c|c|c}
\hline \multirow{2}{*}{ Unidade } & \multicolumn{3}{|c}{ Índice de desempenho inovador } \\
\cline { 2 - 4 } & Espanha & Santa Catarina & Pará \\
\hline Empresa 1 & 0,842 & 0,390 & 0,546 \\
Empresa 2 & 0,576 & 0,813 & 0,781 \\
Empresa 3 & 0,485 & 0,510 & 0,261 \\
Empresa 4 & 0,331 & 0,831 & 0,699 \\
Empresa 5 & 0,996 & 0,942 & 0,240 \\
Empresa 6 & 0,686 & 0,881 & 0,700 \\
Empresa 7 & 0,304 & 0,579 & 0,328 \\
Empresa 8 & 0,258 & 0,494 & 0,466 \\
Empresa 9 & 0,905 & 0,080 & 0,000 \\
Empresa 10 & 0,987 & 0,847 & 0,428 \\
Empresa 11 & - & 0,611 & 0,397 \\
Empresa 12 & - & - & 0,510 \\
Média & 0,637 & 0,634 & 0,446 \\
Mediana & 0,631 & 0,611 & 0,447 \\
\hline
\end{tabular}

Fonte: elaboração dos autores com base em pesquisa de campo, 2012/2013. 
Considerando o processo de hierarquização em valores intervalares, quatro empresas espanholas (40\%) apresentaram IDI alto, portanto, maiores que 7, três apresentaram IDI médio (30\%), entre 0,350 e 0,699 e três (30\%) tiveram baixo desempenho. As empresas catarinenses obtiveram resultados estatísticos aproximados das galegas. Cinco delas (45\%) apresentaram desempenho inovador superior a 7 , enquanto que outras cinco (45\%) apresentaram médio desempenho e apenas uma firma (10\%) obteve fraco desempenho. O Pará teve duas empresas (17\%) com valor igual ou superior a 7 , portanto, com médio desempenho, seis empresas (50\%) com baixo desempenho. Ademais, tanto a média quanto a mediana apontam as empresas da Galícia como as mais inovadoras, seguidas pelas brasileiras do sul do Brasil (Santa Catarina), embora com ligeira aproximação entre as galegas e as catarinenses.

Alguns fatores observados no decorrer desta pesquisa podem ser esclarecedores em relação aos dados e ao fato de as empresas galegas terem obtido melhor resultado estatístico. Primeiramente, existe certo equilíbrio entre as empresas de Santa Catarina e da Espanha, por exemplo, porque as culturas empreendedoras têm semelhanças, além da existência de relações de capital. A maneira de pensar a atividade em termos de gestão organizacional e de competitividade tem características muito comuns entre ambos, embora na prática existam hiatos.

Em ambos os ambientes, a inovação é pensada estrategicamente, como um elemento fundamental no processo de desenvolvimento das empresas e da atividade. As firmas que apresentaram melhor desempenho têm como parte de seus planos o desenvolvimento e/ou a adoção de práticas inovativas tanto em processo quanto em produtos como metas frequentes.

As empresas galegas, mais do que as catarinenses, têm um diferencial que as torna mais fortes, inclusive refletindo sua força para o próprio mercado, tanto no aspecto da concorrência direta quanto para o consumidor em última instância. Trata-se do ambiente externo que as circunda e que, de certo modo, emergiu do próprio ambiente produtivo. Portanto, é um processo endógeno. Trata-se da capacidade institucional presente no ambiente produtivo, no qual as empresas da pesca industrial galegas estão inseridas, assim, tem sido cada vez mais fortificado e com maior capacidade de promover competitividade ao segmento, de tal modo que o diferencia em relação aos demais da Espanha e do Brasil.

Por isso, existe um arranjo institucional robusto capaz de suprir as demandas das empresas em termos de inovação - produto e processo -, nesse caso desenvolvendo mecanismos para melhorar o desempenho das empresas e minimizar os impactos sobre os ambientes naturais em que atuam, permitindo-lhes direcionar 
o máximo de recursos disponíveis (inclusive tempo) para melhorar sua capacidade de competir, fortalecendo-as para o enfrentamento do mercado. Isso tem faltado às empresas brasileiras, pois - elas também - enfrentam um de seus mais atormentados momentos no cenário econômico.

\section{Considerações finais}

A pesca é uma importante atividade socioeconômica com plena capacidade de colaborar para o desenvolvimento dos ambientes em que está inserida, seja na forma direta ou indireta. É perceptível nos casos do Brasil e da Espanha, mais intensamente, a relevância dessa atividade bem como o papel social que ela exerce.

Todavia, como nos ensina a teoria neoschumpeteriana, toda atividade depende da trajetória construída ao longo do tempo em suas mais diversas dimensões. Logo, a pesca cumprirá seu papel de importante instrumento de promoção do desenvolvimento se forem canalizados e aplicados os recursos (políticos, econômicos, institucionais) de modo que haja um processo evolutivo, cuja capacidade produtiva consiga ser traduzida em vantagem social e econômica.

Isso é possível quando existe um compromisso conjunto dos agentes sociais, ou seja, quando suas decisões internas externalizam seus desdobramentos de tal modo que os resultados sejam positivos para todos. Isso foi percebido no ambiente produtivo da pesca industrial da Galícia espanhola e tem sido peça fundamental para o fortalecimento daquele setor frente aos seus potenciais concorrentes.

As ações não são feitas de forma isolada, mas são acessíveis a todos os agentes que desejam fazer parte delas. Por isso, as práticas inovativas no âmbito da pesca industrial galega têm tido bom resultado. As empresas associaram-se às instituições e o feedback as fortaleceu, de modo que as inovações, portanto, fazem parte de suas rotinas. Vão desde a elaboração de novos produtos até orientações de como reduzir custos e impactos ambientais com base em melhores formas de uso e condução dos instrumentos de produção.

Desse modo, as janelas de oportunidade são efetivamente acessadas, embora as dificuldades existam. Porém, diferentemente de Santa Catarina, e mais ainda do Pará, o espírito empreendedor dos agentes tem convergência com os objetivos e as metas das instituições locais e externas. Portanto, mais do que um plano de ideias, existe a união de forças que possibilitam um real imaginário: o desenvolvimento sustentado da pesca industrial galega. 


\title{
The innovative behaviour and effects on production fishing industry in Pará and Santa Catarina states (Brazil) and Spanish galicia
}

\begin{abstract}
This article aimed show the results of a comparative study of industrial fishing activity carried out in two main fish producing States of Brazil - Santa Catarina (South) and Pará (North) and the region of Galicia, Spain's largest fishing warehouse. The main objective was to highlight innovative practices of companies in the sector, especially regarding its role regarding the promotion of the development of the activity and its consequences on their environments. This possibility was achieved using statistical factorial analysis of the instruments that allowed to produce a groundbreaking performance indicator of the surveyed companies, which are classified as more or less innovative. The results showed the Galician companies are as a whole the most innovate reflecting on market behavior. Thereby, it is interesting to understand what makes them have a better performance in relation to Brazil in order to understand why these are not very favourable to competitiveness situation and its own sustainability.
\end{abstract}

Keywords: Innovation. Industrial fishing. Competitiveness. Fishing economy. Institutions.

\section{El comportamiento innovador y los efectos sobre la producción pesquera industrial de los estados de Pará y Santa catarina (Brasil) y de la galícia española}

\section{Resumen}

El objetivo de este artículo es mostrar los resultados de un estudio comparativo de las actividades pesqueras industriales en los dos principales estados productores de pescado en Brasil - Santa Catarina (Sur) y Pará (Norte) y la región de Galicia, la mayor productora de pescado de España. El principal objetivo era destacar las prácticas innovadoras de las empresas del sector, especialmente en relación con su papel en la promoción del desarrollo de la actividad y sus consecuencias en su alrededor. Esto fue posible utilizando las herramientas estadísticas de análisis factorial que establecieron un indicador de los resultados de innovación de las empresas investigadas que las classifica como más o menos innovadoras. Los resultados mostraron las empresas gallegas en su conjunto como más innovadoras reflejado en el comportamiento del mercado. Por lo tanto, hay que conocer los factores que las hacen tener un mejor desempeño frente a las brasileñas con el fin de entender por qué estas últimas están en situación no muy favorable para la competitividad y su propia sostenibilidad.

Palabras clave: Innovación. Pesca industrial. Competitividad. Economía pesquera. Instituciones.

Classificação JEL: B52

Teoria e Evidência Econômica - Ano 21, n. 44, p. 149-164, jan./jun. 2015 


\section{Notas}

1 Porém, deve-se destacar que nem toda ação de Pesquisa e Desenvolvimento efetiva-se em processo, pois, em geral, dependem da viabilidade econômica. A P\&D é condição para se implementar processos inovativos eficientes e duradouros.

2 Para mais detalhes ver Fávero et al. (2009) e Santana, Carvalho e Mendes. (2008). Os dados utilizados foram submetidos ao teste de normalidade de Shapiro (Shapiro-Wilker W) e os mesmos apresentaram distribuição normal.

\section{Referências}

DOSI, G. Mudança técnica e transformação industrial: a teoria e uma aplicação à indústria de semicondutores. Campinas: Unicamp, 2006.

DOSI, G. et al. Technical change and economic theory. London: Pinter Publishers, 1988.

FÁVERO, L. P. et al. Análise de dados: modelagem multivariada para tomada de decisões. Rio de Janeiro: Elsevier, 2009.

KIM, L. Da limitação à inovação: a dinâmica do aprendizado tecnológico da Coréia. Campinas: Unicamp, 2005.

LASTRES, H. M. M. et al. Globalização e inovação localizada. In: LASTRES, H. M. M; ALBAGLI, S. (Org.). Informação e globalização na era do conhecimento. Rio de Janeiro: Campus, 1999. p. 164-190.

LUNDVALL, B.-Ä. (Ed.). National systems of innovation: towards a theory of innovation and interactive leaning. London: Pinter Publishers, 1992.

NELSON, R. R. As fontes do crescimento econômico. Campinas: Unicamp, 2006.

NELSON, R. R.; WINTER, S. Uma teoria evolucionária da mudança econômica. Campinas: Unicamp, 2005.

POSSAS, M. Em direção a um paradigma microdinâmico: a abordagem neoschumpeteriana. In: AMADEO, E. (Org.). Ensaios sobre economia política moderna. São Paulo: Marco Zero, 1989.

ROSENBERG, N. Por dentro da caixa preta: tecnologia e economia. Campinas: Unicamp, 2006.

SACHS, J. D. A riqueza de todos. Rio de Janeiro: Nova Fronteira, 2008.

SANTANA, A. C.; CARVALHO, D. F.; MENDES, F. A. T. Análise sistêmica da fruticultura paraense: organização, mercado e competitividade empresarial. Belém: Basa, 2008.

STALLIVIERI, F.; BRITTO, J. Dinâmica inovativa de empresas inseridas em arranjos produtivos locais: uma análise para casos selecionados em Santa Catarina. In: ENCONTRO NACIONAL DE ECONOMIA POLÍTICA, 14, 2009, São Paulo. Anais... São Paulo, jun. 2009.

SUCASAS, L. F. A. Avaliação do resíduo do processamento de pescado e desenvolvimento de co-produtos visando o incremento da sustentabilidade na cadeia produtiva. 2011. $164 \mathrm{f}$. Tese (Doutorado em Energia Nuclear na Agricultura e no Ambiente) - Centro de Energia Nuclear na Agricultura da Universidade de São Paulo, Piracicaba, 2011. 164 f.

TEECE, D. J. As aptidões das empresas e o desenvolvimento econômico: implicações para as economias de industrialização. In: KIM, L.; NELSON, R. (Org.). Tecnologia, aprendizado e inovação: as experiências das economias de industrialização recente. Campinas: Unicamp, 2005. (Clássicos da Inovação). 